\title{
Inhibition of Heat Shock Protein 90 suppresses TWIST1 Transcription $[$ [
}

\author{
Kay Yi Chong, ${ }^{1}$ Min Kang, ${ }^{1}$ Francesca Garofalo, Daiki Ueno, Huamao Liang, Sarah Cady, \\ Oluwagbemisola Madarikan, Nicholas Pitruzzello, Cheng-Hsiu Tsai, Tobias M.P. Hartwich, \\ Brian M. Shuch, and () Yang Yang-Hartwich
}

Department of Obstetrics, Gynecology, and Reproductive Sciences, Yale School of Medicine, New Haven, Connecticut (K.Y.C., M.K., F.G., S.C., T.M.P.H., Y.Y.-H.); The First Affiliated Hospital of Guangxi Medical University, Nanning, Guangxi, China (M.K.); Ronald Reagan UCLA Medical Center, University of California Los Angeles, Santa Monica, California (D.U., B.M.S.); Department of Obstetrics and Gynecology, Peking University Third Hospital, Beijing, China (H.L.); Department of Biology and Environmental Science, University of New Haven, West Haven, Connecticut (O.M., N.P., C.-H.T.); and Yale Cancer Center, New Haven, Connecticut (Y.Y.-H.)

Received February 21, 2019; accepted June 3, 2019

\begin{abstract}
Molecular chaperone heat shock protein 90 (HSP90) is involved in oncogenic signaling pathways including epithelialmesenchymal transition (EMT), a key process in tumor initiation, progression, metastasis, and chemoresistance. The molecular mechanisms underlying the involvement of HSP90 in EMT are still under investigation. In this study, we identified a previously unrecognized role of HSP90 in cooperating with signal transducer and activator of transcription 3 (STAT3) to regulate TWIST1 transcription in cancer cells. The HSP90 inhibitor $17-\mathrm{N}$-allylamino-17demethoxygeldanamycin suppressed TWIST1 mRNA expression and promoter activity in epithelial ovarian cancer, renal clear cell cancer, and nasopharyngeal cancer cell lines. The interactions between HSP90 and transcription factors were visualized in cancer cell lines and tumor tissues
\end{abstract}

\section{Introduction}

Heat shock protein 90 (HSP90) is an ATP-dependent molecular chaperone with a critical role in maintaining protein homeostasis or proteostasis. Examples of processes mediated by

The work was supported by a Rivkin Center Cookie Laughlin Bridge Award, a Discovery to Cure Ovarian Cancer Research Grant, a Colleen's Dream Foundation Research Grant, the Office of the Assistant Secretary of Defense for Health Affairs through the Ovarian Cancer Research Program [Award W81XWH- 15-1-0221], the National Natural Science Foundation of China [Award 81760542], the Research Foundation of the Science and Technology Department of Guangxi Province, China [Award 2016GXNSFAA380252], the Guangxi Medical University Training Program for Distinguished Young Scholars, and a Medical Excellence Award funded by a Creative Research Development Grant from the First Affiliated Hospital of Guangxi Medical University.

The authors declare no potential conflicts of interest.

${ }^{1}$ K.Y.C. and M.K. contributed equally to the work.

https://doi.org/10.1124/mol.119.116137.

S This article has supplemental material available at molpharm.aspetjournals.org. using proximity ligation assays. Our findings reveal that HSP9O promotes the binding of STAT3 to the TWIST1 promoter, leading to the transcription of TWIST1. The inhibition of HSP9O downregulates STAT3 activity and TWIST1 transcription, thereby suppressing EMT and potentially inhibiting tumor progression, metastasis, and chemoresistance in different types of cancers.

\section{SIGNIFICANCE STATEMENT}

Our study provides new evidence that HSP9O promotes EMT through enhancing TWIST1 transcription, which can be suppressed by HSP90 inhibitors. The HSP90 inhibitor inhibits EMT, thus potentially slowing down tumor growth, invasion, dissemination, metastasis, and drug resistance. These findings will hopefully pave the way for new therapeutic opportunities to target EMT and metastasis using HSP90 inhibitors.

ABBREVIATIONS: 17-AAG, 17-N-allylamino-17-demethoxygeldanamycin; ChIP, chromatin immunoprecipitation; 3D, three-dimensional; EMT, epithelial-mesenchymal transition; GAPDH, glyceraldehyde-3-phosphate dehydrogenase; HIF-1, hypoxia-inducible factor 1; HSP90, heat shock protein 90; IF, immunofluorescence; IL-6, interleukin-6; IP, immunoprecipitation; PLA, proximity ligation assay; Ser727, serine 727; STAT, signal transducer and activator of transcription; Tyr705, tyrosine 705. 
oncogenic signaling pathways, inhibiting HSP90 has become a major focus in drug development efforts. HSP90 inhibitors have shown promise in clinical trials for cancer treatment; however, several problems remain unresolved such as drug toxicity and limited efficacy (Wang et al., 2016). The optimal use of HSP90 inhibitors for therapy requires thorough understanding of the relationship between HSP90 and its client proteins along with the mechanisms by which HSP90 modulates protein function and activity.

TWIST1 is a basic helix-loop-helix transcription factor and a key driver of epithelial-mesenchymal transition (EMT). TWIST1 transcriptionally represses E-cadherin and other cell-cell adhesion molecules, leading to the loss of the epithelial phenotype (Yang et al., 2004). TWIST1 has important functions in embryonic development and tumor metastasis, the latter of which involves tumor cells undergoing EMT to gain invasive and migratory properties (Qin et al., 2012). HSP90 has been speculated to regulate EMT; however, it is unclear whether HSP90 directly regulates TWIST1 in cells (Hance et al., 2012).

Our study provides insight into how HSP90 promotes the transcription and expression of TWIST1, thus promoting EMT. We hypothesize that HSP90 enhances the transcription of TWIST1 through interacting with its client proteins; in particular, with signal transducer and activator of transcription (STAT) 3. We tested our hypothesis in ovarian, renal, and nasopharyngeal cancer cell lines. These cancer types are anatomically, genetically, and phenotypically different. They are also clinically treated with different therapeutic agents. However, these cancers commonly maintain high levels of HSP90 and/or constitutively activated STAT3 (Huang et al., 2000; Guo et al., 2009; Cheng et al., 2018). TWIST1 and EMT have been shown to play key roles in the progression and drug resistance in these cancers (Davidowitz et al., 2014; Zhuo et al., 2014; Singla et al., 2018). Using these cancer cell lines in our study allows us to understand the pharmacological actions of HSP90 inhibitors at the molecular level in different types of cancers with respect to their ability to inhibit EMT, metastasis, and chemoresistance.

\section{Materials and Methods}

Reagents and Cell Lines. 17- $N$-allylamino-17-demethoxygeldanamycin (17-AAG), NSC74859, and nifuroxazide were purchased from APEXBIO (Boston, MA). The compounds were dissolved in DMSO at $10 \mathrm{mM}$ and diluted with cell culture medium to the desired concentrations. TWIST1 promoter plasmid was provided by Dr. Carlotta A. Glackin (City of Hope Beckman Research Institute, Duarte, CA). It was constructed by cloning the TWIST1 promoter (1 kilobase upstream of the coding start site of the Twist1 gene) into the pGL3 vector. The pGL3 vector and pRL-TK Renilla luciferase control reporter vector were purchased from Promega (Madison, WI). HSP90 plasmid was purchased from transOMIC technologies (Huntsville, AL).

Ovarian cancer cell line SKOV3 and renal cancer cell line A498 were obtained from American Type Culture Collection (Manassas, VA). The RCC4 cell line was purchased from Sigma-Aldrich (St. Louis, MO). OVCAR8 cells were provided by Dr. Anirban Mitra (Indiana University School of Medicine, Indianapolis, IN). A2780 cells and patientderived ovarian cancer cell lines OVC201, OVC203, OVC205, and OVC302 were provided by Dr. Gil Mor (Yale University School of Medicine). The patient-derived cell lines were isolated from tumor samples of patients diagnosed with serous epithelial ovarian cancer. All samples used for establishing the cell lines in this study were collected after obtaining patient consent and approval from the
Human Investigation Committee at Yale University School of Medicine. Two nasopharyngeal carcinoma cell lines, C666-1 and CNE-1, were obtained from the Chinese Academy of Sciences Cell Bank. Cell lines were authenticated by short tandem repeat DNA profiling.

Cell Culture. Ovarian and renal cancer cell lines were propagated in RPMI 1640 medium containing 10\% FBS, L-glutamine, and penicillin/streptomycin. Nasopharyngeal cancer cell lines were cultured in Dulbecco's modified Eagle's medium containing 10\% FBS, L-glutamine, and penicillin/streptomycin. They were all cultured in an incubator at $37^{\circ} \mathrm{C}\left(5 \% \mathrm{CO}_{2}\right)$.

For the three-dimensional (3D) culture experiments, cancer cells were seeded in 96-well ultralow attachment plates with a cell-repellent surface (Greiner Bio-One, Monroe, NC) at a density of 1000 cells/well. Ovarian and nasopharyngeal cancer cells were cultured in 3D Tumorsphere Medium XF (PromoCell, Heidelberg, Germany). Renal cancer cell lines were cultured in Opti-MEM Reduced Serum Medium with no phenol red (Life Technologies, Carlsbad, CA).

Wound Healing Assay. Cells were plated in a 24-well plate (50,000 cells per well). After 24 hours, the confluent cells were wounded by pipette tips using a semimanual wound maker tool. The wells were washed with PBS three times before new growth medium with or without 17-AAG $(0.2 \mu \mathrm{M})$ was added to the wells. The wound was imaged using a digital microscope cell imager. The wound width was recorded at different time points. The wound closure rate was calculated as [(initial width - current width)/initial width]\%.

Quantitative Real-Time Polymerase Chain Reaction. Total RNA was extracted using the Total RNA Purification Kit (Norgen Biotek, Thorold, ON, Canada). The qScript cDNA SuperMix Kit was used to synthesize cDNA (Quantabio, Beverly, MA). Quantitative polymerase chain reaction was performed using SYBR Green Supermix (Bio-Rad, Hercules, CA). The CFX Connect qPCR Detection System (Bio-Rad) was used to detect the SYBR Green signals. Glyceraldehyde-3-phosphate dehydrogenase (GAPDH) was used as the reference gene. Relative expression was calculated using the comparative $\Delta \Delta \mathrm{CT}$ method. All reactions were performed with three biologic replicates, and each replicate included three technical replicates. The primer sequences are as follows: Twist-sense $5^{\prime}$ GTCATGGCCAACGTGCGGGA-3'; Twist-antisense 5'-GCCGCCAGCTTGAGGGTCTG-3'; GAPDH-sense 5'-AATGAAGGGGTCATTGATGG-3'; and GAPDH-antisense 5'-AAGGTGAAGGTCGGAGTCAA-3' .

Western Blot. Cell lysates were prepared using cell lysis buffer ( $1 \%$ Triton X-100, 0.05\% SDS, $100 \mathrm{mM} \mathrm{Na}_{2} \mathrm{HPO}_{4}$, and $150 \mathrm{mM} \mathrm{NaCl}$ ). Then, $10 \mu \mathrm{g}$ of each protein lysate was electrophoresed on $12 \%$ SDS-polyacrylamide gel and transferred onto Amersham Hybond 0.45 polyvinylidene difluoride membranes (GE Healthcare, Chicago, IL). After incubation in blocking buffer ( $5 \% \mathrm{w} / \mathrm{v}$ bovine serum albumin, $1 \mathrm{X}$ Tris-buffered saline, and $0.1 \%$ Tween-20), the membranes were incubated with primary antibodies at $4^{\circ} \mathrm{C}$ overnight followed by secondary antibodies for 1 hour at room temperature. The blots were developed using Clarity or Clarity Max Western ECL Blotting Substrates (Bio-Rad). Antibodies were obtained from Cell Signaling Technology (Danvers, MA). The antibody product numbers and dilutions are as follows: anti- $\beta$-catenin (\#8480, 1:1000), anti-hypoxia-inducible factor 1 (anti-HIF-1) $\alpha$ (\#36169, 1:1000), anti-HIF-1 $\beta$ (\#5537, 1:1000), anti-STAT3 (\#4904, 1:1000), anti-STAT5 (\#94205, 1:1000), anti-STAT6 (\#5397, 1:1000), anti-N-Myc (\#9405, 1:1000), and anti-MyoD1 (\#13812, 1: 1000). Horseradish peroxidase-conjugated anti-GAPDH antibody (\#HRP-60004), goat anti-mouse IgG (\#SA00001-1), and goat anti-rabbit IgG (\#SA00001-2) were purchased from Proteintech (Rosemont, IL).

Proximity Ligation Assay. The tissue microarrays used in this study were purchased from US Biomax, Inc. (Derwood, MD). The formalin-fixed paraffin-embedded tumor tissue sections were deparaffinized and hydrated by three washes of xylene for 5 minutes each, followed by two washes of $100 \%$ ethanol for 10 minutes each, two washes of $95 \%$ ethanol for 10 minutes each, and two washes of $\mathrm{ddH}_{2} \mathrm{O}$ for 5 minutes each. Slides were submersed in $1 \mathrm{X}$ citrate unmasking solution and heated in a microwave until the solution began to boil, followed by 10 -minute incubation at $95-98^{\circ} \mathrm{C}$. After cooling, the slides 
were incubated in blocking buffer ( $5 \%$ normal serum and $0.3 \%$ Triton $\mathrm{X}-100$ in $1 \mathrm{X}$ PBS) for 60 minutes at room temperature. Cultured cells were cultured on chamber slides, fixed with $4 \%$ paraformaldehyde in PBS for 10 minutes at room temperature, and then washed with PBS three times followed by incubation in blocking buffer. Proximity ligation assay (PLA) was performed according to the protocol provided by the manufacturer of the Duolink In Situ Red Starter Kit (\#DUO92101, Simga-Aldrich). Briefly, mouse anti-HSP90 antibody (\#SC-515081, Santa Cruz Biotechnology, Dallas, TX) and rabbit anti-STAT3 antibody (\#A302405A, Bethyl Laboratories, Montgomery, TX) were diluted in antibody diluent and added to the samples. After overnight incubation at $4^{\circ} \mathrm{C}$, slides were washed three times with $1 \mathrm{X}$ PBS. Two PLA probes were diluted 1:5 and incubated with samples for 60 minutes at $37^{\circ} \mathrm{C}$. After washing with $1 \mathrm{X}$ wash buffer $\mathrm{A}$ twice for 5 minutes, the ligation mix was added to the slides to incubate for 30 minutes at $37^{\circ} \mathrm{C}$. After washing with $1 \mathrm{X}$ wash buffer A twice for 2 minutes, the amplification mix was added to the slides and left to incubate for 100 minutes at $37^{\circ} \mathrm{C}$. The slides were washed with $1 \mathrm{X}$ wash buffer $\mathrm{B}$ twice for 10 minutes and $0.01 \mathrm{X}$ wash buffer B for 1 minute. Duolink In Situ Mounting Medium with 4,6-diamidino2-phenylindole was used to mount the samples for 15 minutes. The slides were analyzed using a confocal microscope (Leica TCS SP8; Leica Microsystems, Wetzlar, Germany).

Immunofluorescence Staining. Formalin-fixed paraffin-embedded tumor tissue sections and cultured cells for immunofluorescence (IF) staining were prepared according to the same protocol used for the PLA. The primary antibody was diluted in antibody dilution buffer ( $1 \%$ bovine serum albumin and $0.3 \%$ Triton X-100 in $1 \mathrm{X}$ PBS) and added to the slides followed by incubation overnight at $4^{\circ} \mathrm{C}$. After rinsing three times in $1 \mathrm{X}$ PBS for 5 minutes each, the fluorochrome-conjugated secondary antibody (diluted in antibody dilution buffer) was added and incubated for 1 hour at room temperature in the dark. Prolong Gold Antifade Reagent with 4,6-diamidino-2-phenylindole was used to mount the slides (\#9071; Cell Signaling Technology). The antibody dilutions are as follows: anti$\beta$-catenin (\#8480, 1:100; Cell Signaling Technology), anti-HIF-1 $\alpha$ (\#36169, 1:1000; Cell Signaling Technology), anti-HIF-1 $\beta$ (\#5537, 1 : 100; Cell Signaling Technology), anti-STAT3 antibody (\#A302405A; Bethyl Laboratories), anti-STAT5 (\#94205, 1:100; Cell Signaling Technology), anti-HSP90 (\#SC-515081, 1:100; Santa Cruz), and Alexa Fluor 594 conjugated anti-mouse or anti-rabbit IgG (\#8890 or \#8889, 1:1000; Cell Signaling Technology).

Nuclear Protein Fraction Extraction. Nuclear and cytoplasmic fractions were extracted using the NE-PER Nuclear and Cytoplasmic Extraction Reagent Kits (Thermo Scientific, Rockford, IL) according to the manufacturer's protocol.

Chromatin Immunoprecipitation. Chromatin immunoprecipitation (ChIP) was performed using the Zymo-Spin ChIP Kit (Zymo Research, Irvine, CA) and SureBeads Magnetic Beads (Bio-Rad) according to each manufacturer's protocol. Anti-STAT3 antibody (Cell Signaling Technology) was added to the test groups and mouse IgG (Santa Cruz) was added to the control groups. Quantitative polymerase chain reaction was performed to measure TWIST1 DNA levels. Signals obtained from the immunoprecipitation (IP) STAT3 and IgG control samples were normalized to that of the input control samples. After normalization, signals of the IP STAT3 samples were divided by that of the IgG control samples to obtain the fold increase in STAT3 binding to TWIST1 relative to the background signal.

Coimmunoprecipitation. SureBeads (Bio-Rad) were washed three times with PBS-Tween 20 buffer (PBS $+0.1 \%$ Tween 20). Antibody $(1 \mu \mathrm{g})$ was added to the beads in a final volume of $200 \mu \mathrm{l}$ and rotated 10 minutes at room temperature. The beads were washed three times with PBS-Tween 20 buffer before the antibody-conjugated beads were ready for IP. Cell lysate was added to the beads and rotated for 1 hour at room temperature. The beads were magnetized to discard the supernatant. After the beads were thoroughly washed three to five times with PBS-Tween 20, the resuspended beads were transferred to a new tube. The beads were magnetized and the residual buffer was aspirated from the tube. Laemmli buffer $(40 \mu \mathrm{l})$ was added to the tube and incubated for 5 minutes at $90^{\circ} \mathrm{C}$. Finally, the beads were magnetized to move the eluent to a new tube. The eluent was the IP product for the western blot tests. Antibodies were obtained from Cell Signaling Technology (anti-STAT3, \#4904) and Santa Cruz Biotechnology (anti-HSP90, \#SC-515081).

TWIST1 Promoter Luciferase Assay. Cells were cotransfected with pGL3-Twist1 promoter plasmid and pRL-TK plasmid in the presence of HSP90 or green fluorescence protein (GFP) plasmid using ViaFect Transfection Reagent (Promega) according to the manufacturer's instructions. Transfected cells were treated with drugs 24 hours post-transfection. The cells were collected after 24-hour treatment. Luciferase activity was measured using the Dual-Luciferase Reporter Assay Kit and GloMax Navigator Luminometer System (Promega) according to the manufacturer's instructions. The relative Firefly luciferase activity was calculated by normalizing to the Renilla luciferase activity.

EMT Antibody CellSimple Assays. The CellSimple Epithelial to Mesenchymal Transition Antibody Assay Kit (Cell Signaling Technology) was used on the CellSimple Cell Analyzer (Cell Signaling Technology) to measure vimentin expression in cells according to the manufacturer's protocol. Briefly, cells were fixed, permeabilized, stained for vimentin using a phycoerythrin-conjugated antibody, and allowed to flow through CellSimple cassettes based on the Coulter principle. The mean fluorescence intensity was measured in the red channel (561 nm), which corresponds to vimentin expression.

Phospho-STAT3 Multiplex Bead CellSimple Assays. The CellSimple Multiplex Bead Assay Kit (Cell Signaling Technology) was used on the CellSimple Cell Analyzer to measure the levels of STAT3 tyrosine 705 (Tyr705) and serine 727 (Ser727) phosphorylation according to the manufacturer's protocol. Briefly, whole cell lysates were incubated with a multiplex bead cocktail followed by a biotinylated detection antibody cocktail. Streptavidin-linked phycoerythrin secondary antibody was then used to detect the antibodies bound to p-STAT3 ${ }^{\text {Tyr705 }}$ and p-STAT3 ${ }^{\text {Ser727 }}$ simultaneously.

Statistical Analysis. Numerical values are presented as the mean \pm S.D. For comparisons between groups, $P$ values were calculated using paired two-tailed one- or two-way ANOVA tests. Values of $P<0.05$ were considered statistically significant. The method of statistical analysis is also specified in the figure legends.

\section{Results}

HSP90 Inhibition Reduces TWIST1 Expression in Cancer Cells. Nonadherent 3D spheroid assays are commonly used to evaluate the activity of normal or cancer stem cells as well as the progression of EMT (Bahmad et al., 2018). We cultured cancer cells in nonadherent plates to promote spheroid formation and evaluated the effects of HSP90 inhibition on spheroid formation ability. We used a smallmolecule HSP90 inhibitor (17-AAG) to treat two epithelial ovarian cancer cell lines (A2780 and SKOV3), two clear cell renal carcinoma cell lines (RCC4 and A498), and two nasopharyngeal carcinoma cell lines (C666-1 and CNE-1). We observed that 17-AAG significantly decreased the number of spheroids formed by these cancer cell lines in a dose-dependent manner (Fig. 1, A and B; Supplemental Fig. 1). This observation suggests that these cancer cells depend on HSP90 function to survive and form tumor spheroids in 3D assays.

To further investigate how HSP90 may be involved in promoting EMT, we assessed the effects of 17-AAG on cell migration ability, a key feature of the mesenchymal phenotype. We found that 17-AAG significantly impaired the wound closure ability of A498, C666-1, and OVC203 cell lines, the latter of which is a patient-derived ovarian cancer cell line (Fig. 1, C-E). These results indicate that HSP90 may be involved in the maintenance of the EMT program in cancer cells. 

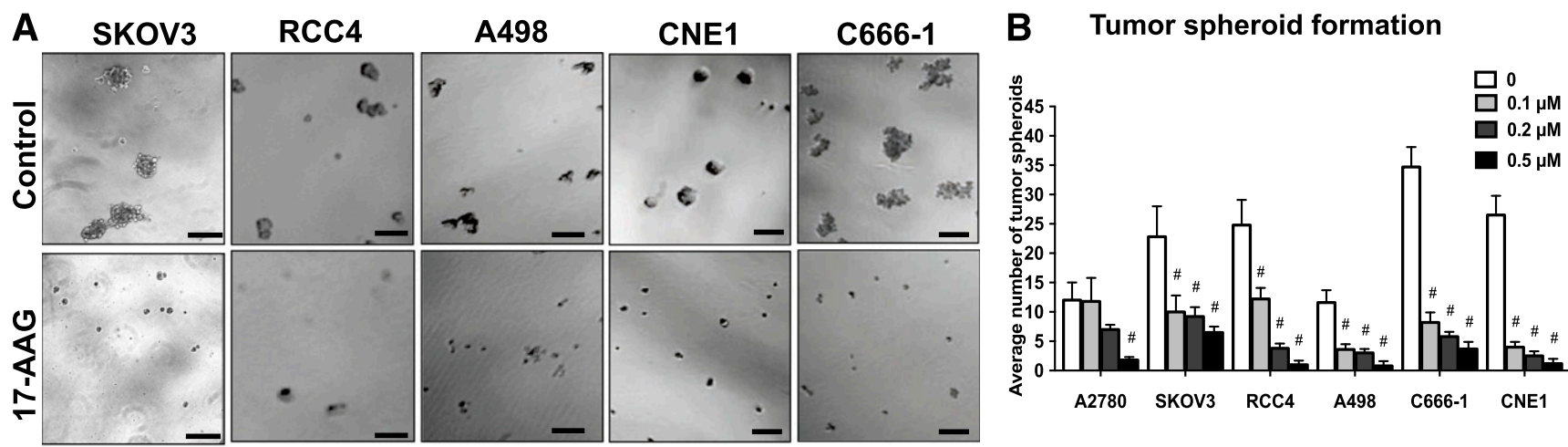

C

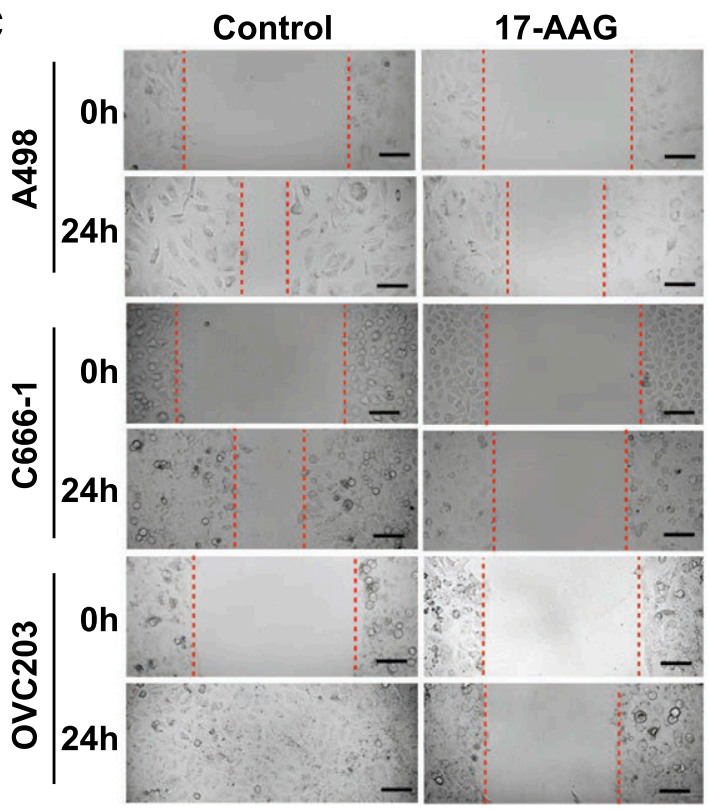

$\mathbf{G}$

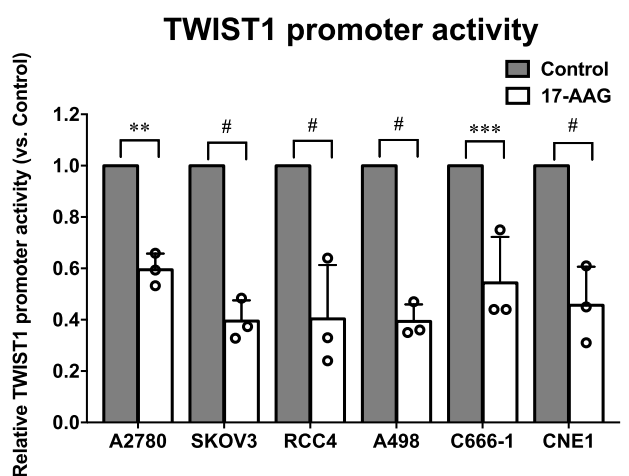

D

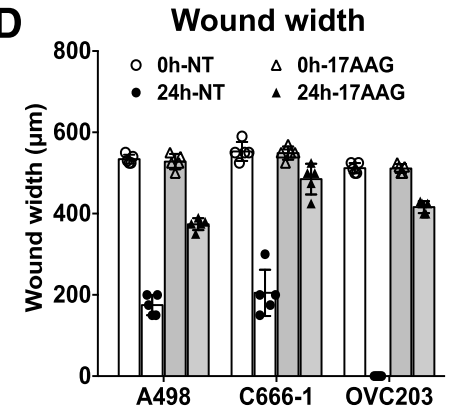

E Wound closure rate (24h)

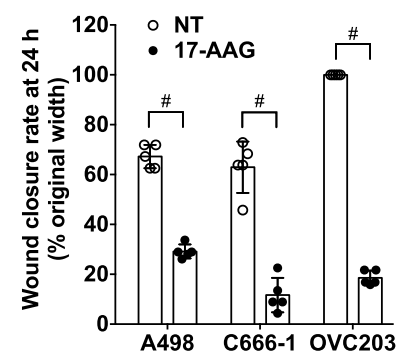

F TWIST1 mRNA expression

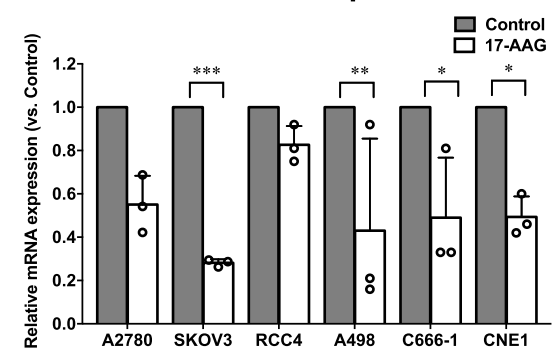

H

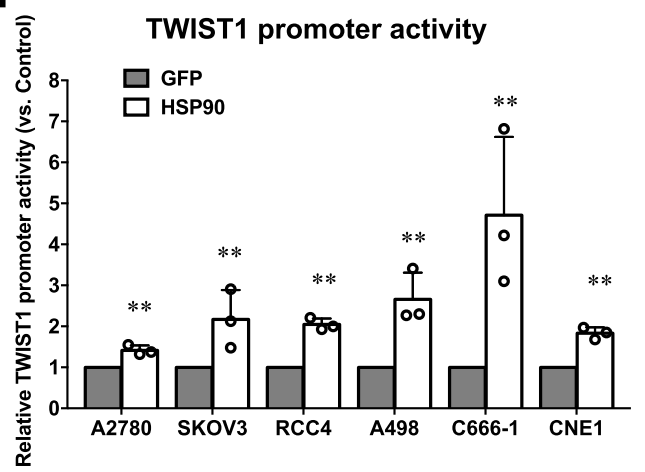

Fig. 1. Effects of HSP90 inhibition on tumor spheroid formation and TWIST1 expression. (A) Representative images of spheroids formed by cancer cell lines after treatment of cells with $0.1 \%$ DMSO (control) or $0.5 \mu \mathrm{M}$ 17-AAG for 96 hours. Scale bar, $100 \mu \mathrm{m}$. (B) The average numbers $(n=6)$ of spheroids formed by cancer cell lines after 17-AAG treatment of 96 hours at the doses indicated. (C) Representative images of wound healing assay before (0 hours) and after (24 hours) treatment of cells with $0.1 \%$ DMSO (control) or $0.2 \mu \mathrm{M} 17$-AAG. Scale bar, $100 \mu \mathrm{m}$. The dotted lines indicate the wound borders. (D) Wound width at 0 and 24 hours of treatment. (E) Wound closure rate at 24 hours calculated based on the wound width data. (F) TWIST1 mRNA levels were determined by quantitative polymerase chain reaction in cancer cell lines treated with either DMSO or $0.5 \mu$ M 17 -AAG for 24 hours. GAPDH was used as the reference gene and the mRNA expression levels are normalized to the control. (G) TWIST1 promoter activity determined by luciferase reporter assays in cancer cell lines treated with DMSO or $0.5 \mu \mathrm{M}$ 17-AAG for 24 hours. Values indicate TWIST1 promoter activity (firefly luminescence) relative to Renilla luciferase internal control. (H) Relative TWIST1 promoter activity determined by luciferase reporter assays in cells transiently overexpressing either GFP or HSP90. All values (mean \pm S.D.) represent three independent experiments if not specified. Each experiment was performed in triplicate. Two-way ANOVA test was followed by Tukey's honestly significant difference test. ${ }^{*} P<0.0001 ;{ }^{* * *} P<0.0005 ;{ }^{* *} P<0.005 ;{ }^{*} P<0.05$. 
We sought to assess whether HSP90 may regulate the expression of genes associated with EMT induction. We noted downregulation of EMT marker gene expression after $0.5 \mu \mathrm{M}$ 17-AAG 24-hour treatment, such as TWIST1 and SLUG, along with an increase in expression of the epithelial marker E-cadherin (Fig. 1F; Supplemental Fig. 2). The mRNA expression of TWIST1 significantly decreased after 17-AAG treatment in six cancer cell lines (Fig. $1 \mathrm{~F}$ ). By measuring caspase 3 activity, we found that $0.5 \mu \mathrm{M}$ 17-AAG treatment of 24 hours did not significantly activate apoptosis in most of these cancer cell lines (Supplemental Fig. 3).

To investigate whether the downregulation of TWIST1 expression after 17-AAG treatment is related to a reduction in TWIST1 transcription, we measured the promoter activity of TWIST1 using luciferase reporter assays. TWIST1 promoter activity substantially decreased after $0.5 \mu \mathrm{M}$ 17-AAG treatment of 24 hours (Fig. 1G). Conversely, the overexpression of HSP90 through transient transfection led to significant induction in TWIST1 promoter activity (Fig. 1H). These observations indicate that HSP90 may have a role in promoting the transcription of TWIST1.

HSP90 Interacts with Transcription Factors that Regulate TWIST1 Gene Expression. To understand how 17-AAG suppresses TWIST1 gene expression, we hypothesized that TWIST1 transcription is promoted by one or more transcription factors chaperoned by HSP90. We performed a literature search and shortlisted eight HSP90 client proteins that have been reported to regulate TWIST1 transcription in human cells, namely, $\beta$-catenin, HIF- $1 \alpha$, HIF- $1 \beta$, STAT3, STAT5, STAT6, N-Myc, and MyoD1 (Howe et al., 2003; Cheng et al., 2008; Yang et al., 2008; Koutalianos et al., 2015; Selmi et al., 2015; Zhao et al., 2017; Chen et al., 2018). Using ovarian cancer cell lines A2780, SKOV3, and OVCAR8, we investigated the possibility that these transcription factors may depend on HSP90 to regulate TWIST1 expression.

We performed western blotting and IF staining to identify the baseline levels of these transcription factors in ovarian cancer cell lines. Then, we shortlisted $\beta$-catenin, HIF- $1 \alpha$, HIF- $1 \beta$, and STAT3 for further investigation since these were present in the cells at higher levels (Fig. 2, A and B). We performed PLA to visualize the in situ protein-protein interactions between HSP90 and its client proteins in ovarian cancer cells. We observed that HSP90 interacts with $\beta$-catenin, HIF- $1 \alpha$, HIF- $1 \beta$, and STAT3 in the cytoplasm and nuclei (Fig. 2C). Interestingly, we noted that HSP90/HIF-1 $\alpha$ interactions were mainly localized in the cytoplasm (Fig. 2C), while IF staining of HIF- $1 \alpha$ showed concentrated protein expression in the nuclei (Fig. 2B).

17-AAG Inhibits Interactions between HSP90 and Client Proteins in the Cytoplasm and Nucleus. The nuclear localization of HSP90 in cancer cells has previously been reported (Diehl et al., 2009; Su et al., 2016). We detected the presence of HSP90 in the nuclei of SKOV3 cells by IF staining (Fig. 3A). We also fractionated ovarian cancer cells into cytoplasmic and nuclear protein fractions and confirmed the presence of nuclear HSP90 (Fig. 3B).

We hypothesized that HSP90 regulates TWIST1 transcription by interacting with transcription factors in the nucleus and cytoplasm to promote their stability and transcriptional activity, and 17-AAG may, therefore, inhibit this interaction. To test our hypothesis, we performed PLA to visualize the effects of 17-AAG on the interactions between HSP90 and its client proteins in situ. 17-AAG treatment of 24 hours massively diminished HSP90 interactions with $\beta$-catenin, HIF- $1 \alpha$, HIF-1 $\beta$, and STAT3 in the cytoplasm and nuclei of SKOV3 cells (Fig. 3C; Supplemental Fig. 4).

HSP90 Regulates Protein Stability and Activity of Transcription Factors. We found that 17-AAG treatment of 48 hours significantly decreased the protein levels of $\beta$-catenin, STAT3, and HIF-1 $\alpha$ (Fig. 3D). This observation may reflect the well-established chaperone roles of HSP90 in preserving protein integrity and stability. We reasoned that the inhibition of HSP90 by 17-AAG may lead to the degradation of $\beta$-catenin, STAT3, and/or HIF- $1 \alpha$, which may contribute to the reduction in TWIST1 transcription (Fig. 1, F and G).

However, we noted that a shorter 17-AAG treatment of 24 hours did not decrease the protein levels of the transcription factors (Fig. 3D). Having observed a substantial decrease in TWIST1 transcription in cells treated with 17-AAG for 24 hours (Fig. 1, F and G), we expected the same 24-hour treatment to decrease the stability of the transcription factors. These results led us to hypothesize that apart from maintaining the protein stability of transcription factors, HSP90 may also promote their transcriptional activity. To test our hypothesis,we investigated whether 17-AAG prevented HSP90 from promoting the transcriptional activity of $\beta$-catenin, STAT3, or HIF- $1 \alpha$ in the first 24 hours of treatment before their protein levels were decreased.

We focused our study on STAT3, which has been reported to induce TWIST1 transcription and promote tumor progression, chemoresistance, and metastasis (Wu et al., 2016). We performed a ChIP assay to assess how 17-AAG treatment influences the transcriptional activity of STAT3. We demonstrated that 17-AAG significantly decreased the direct binding of STAT3 to the TWIST1 promoter in A2780 and SKOV3 cells (Fig. 3E). Our data indicate that HSP90 enhances the transcriptional activity of STAT3 by promoting the binding of STAT3 to the TWIST1 promoter. Therefore, the inhibition of HSP90 decreases both the transcriptional activity and protein stability of STAT3.

Using coimmunoprecipitation assay, we confirmed that 17-AAG treatment inhibited the interaction between STAT3 and HSP90 at an early time point. We immunoprecipitated HSP90 protein in the nasopharyngeal carcinoma cell line C666-1 and the patient-derived serous epithelial ovarian cancer cell line OVC203. STAT3 was detected in the coimmunoprecipitation products (Fig. $3 \mathrm{~F}$ ), which confirmed the interaction of HSP90 and STAT3. When both cell lines were treated with $0.5 \mu \mathrm{M}$ 17-AAG for 8 hours, the interactions were significantly inhibited (Fig. 3F). This result also suggests that their interactions were interrupted as early as at 8 hours of 17-AAG treatment before the degradation of STAT3 was observed.

STAT3 is transcriptionally activated by its phosphorylation at $\mathrm{Tyr}^{705}$ or $\mathrm{Ser}^{727}$ residues, leading to different biologic functions (Schuringa et al., 2000). We further assessed the effects of 17-AAG treatment on the phosphorylation of STAT3 at both phosphorylation sites. To better quantify the phosphorylation of STAT3 at both sites simultaneously, we used a bead-based two-plex antibody assay. In this assay, two specific antibodies are covalently linked to a specific-sized magnetic bead to capture the $\mathrm{Tyr}^{705}$ - and $\mathrm{Ser}^{727}$-phosphorylated STAT3 separately. Similar to the sandwich immunoassay principle, samples are incubated with beads followed by a biotinylated detection antibody and streptavidin-linked phycoerythrin. 
A

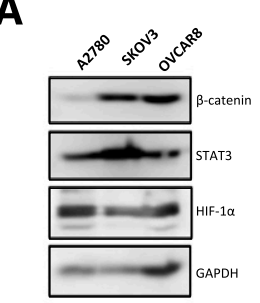

B
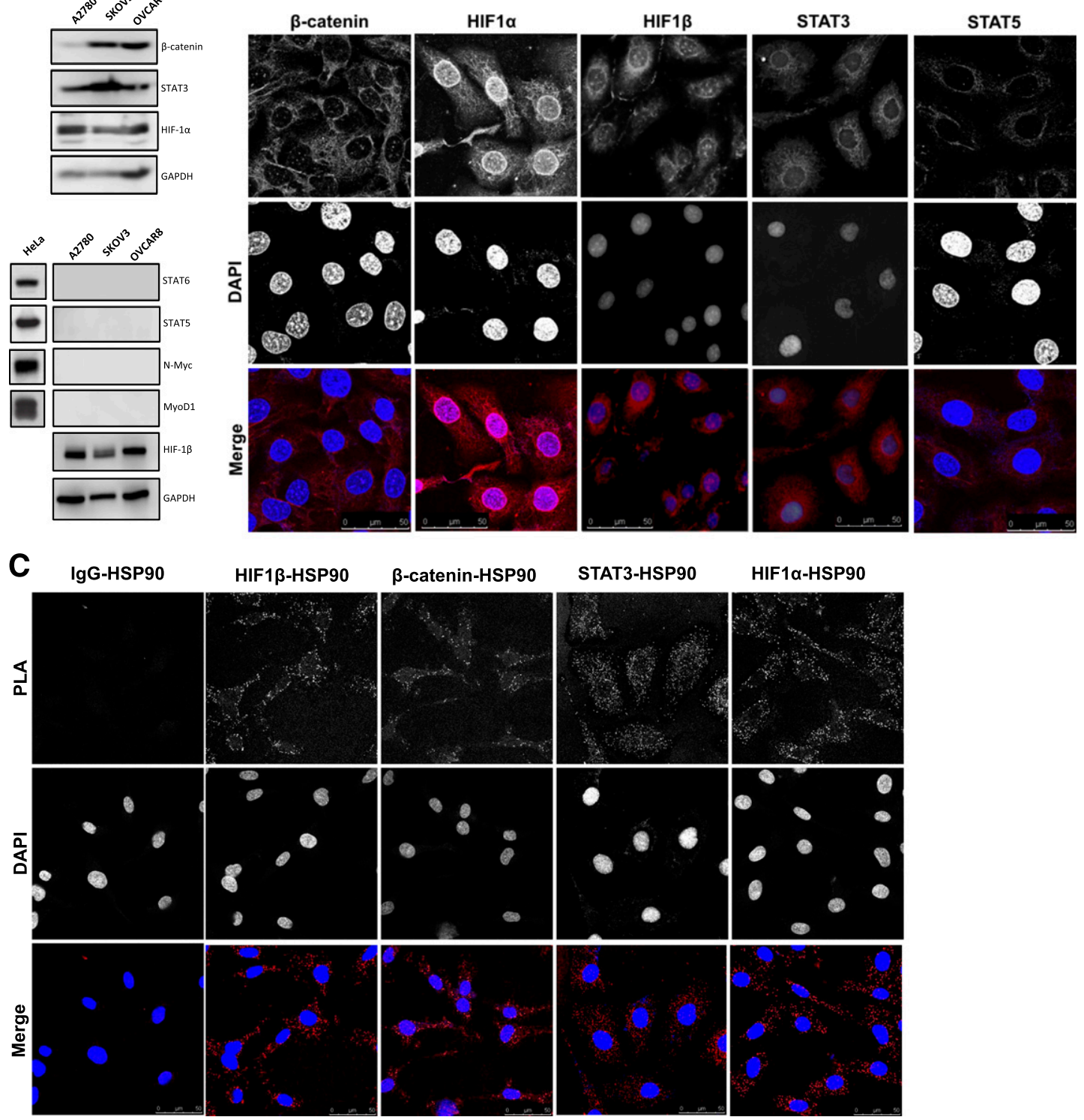

Fig. 2. Protein-protein interactions between HSP90 and transcription factors. (A) Endogenous protein levels of transcription factors in ovarian cancer cell lines detected by western blotting. GAPDH was used as the loading control. HeLa cells served as the positive control for STAT6, STAT5, $\mathrm{N}-\mathrm{Myc}$, and MyoD1. (B) Representative immunofluorescence images of SKOV3 cells stained for the indicated transcription factors (red), nuclear staining [4,6-diamidino-2-phenylindole (DAPI), blue], and merged images. (C) Representative images of in situ PLAs performed using antibodies specific for HSP90, $\beta$-catenin, STAT3, and HIF- $1 \alpha$ in SKOV3 cells. The distinct dots represent the interaction between HSP90 and each transcription factor. Normal IgG control antibody was included as the negative control. Scale bar, $50 \mu \mathrm{m}$.

Using a flow cytometer, we were able to quantify the levels of STAT3 phosphorylation at $\mathrm{Tyr}^{705}$ and $\mathrm{Ser}^{727}$ simultaneously with improved sensitivity compared with using the western blot semiquantification method. We found that the inhibition of HSP90 for 24 hours led to a slight decrease in STAT3 phosphorylated at $\mathrm{Tyr}^{705}$ and a more significant decrease in STAT3 phosphorylated at $\operatorname{Ser}^{727}$ (Fig. 4A).

HSP90 is involved in interleukin-6 (IL-6)-mediated STAT3 activation (Sato et al., 2003). We treated SKOV3 cells with 17-AAG for 6 hours followed by IL-6 stimulation for 10 minutes, and observed a significant decrease in STAT3 phosphorylation at $\mathrm{Ser}^{727}$ (Fig. 4B). IL-6 did not promote phosphorylation at $\mathrm{Tyr}^{705}$ and neither did 17-AAG affect the $\mathrm{Tyr}^{705}$ site (Supplemental Fig. 5). These observations suggest that STAT3 activation is dependent on HSP90 through phosphorylation at $\mathrm{Ser}^{727}$.
IL-6 and transforming growth factor $\beta$ are associated with EMT in diverse cancer types and have been shown to induce EMT in cancer cell lines in vitro (Liu et al., 2014). We treated SKOV3 cells with IL-6 and transforming growth factor $\beta 1$ for 2 weeks to induce EMT, as indicated by an increase in the EMT marker vimentin (Fig. 4, C and D). We found that 17-AAG significantly suppressed vimentin expression at the mRNA and protein levels (Fig. 4, C and D). Since STAT3 is activated by IL-6, these findings strengthen our theory that STAT3 activation contributes to the induction of EMT, and this is dependent on HSP90 function.

HSP90 Mediates TWIST1 Transcription through STAT3 Activation. To examine whether STAT3 is an important player in HSP90-regulated TWIST1 transcription, we used 
A

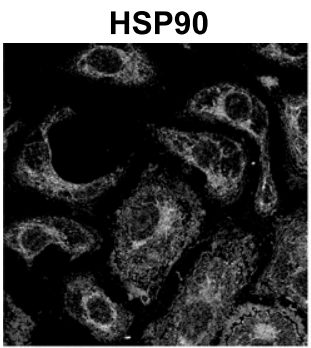

C

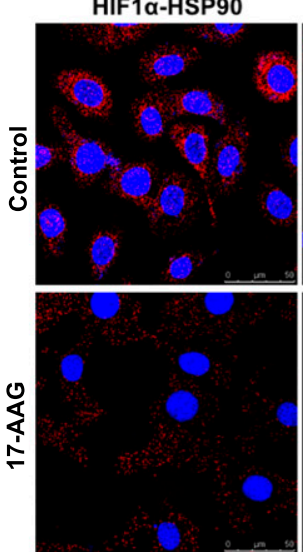

D

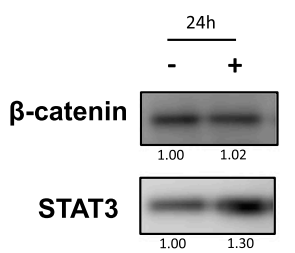

HIF-1 $1 \alpha$

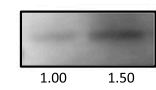

$\beta$-actin

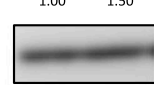

SKOV3
DAPI

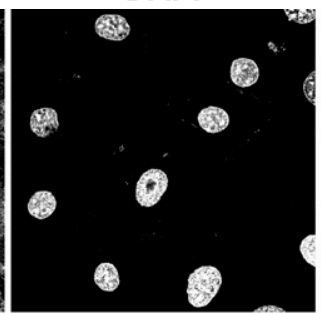

$\beta$-catenin-HSP90
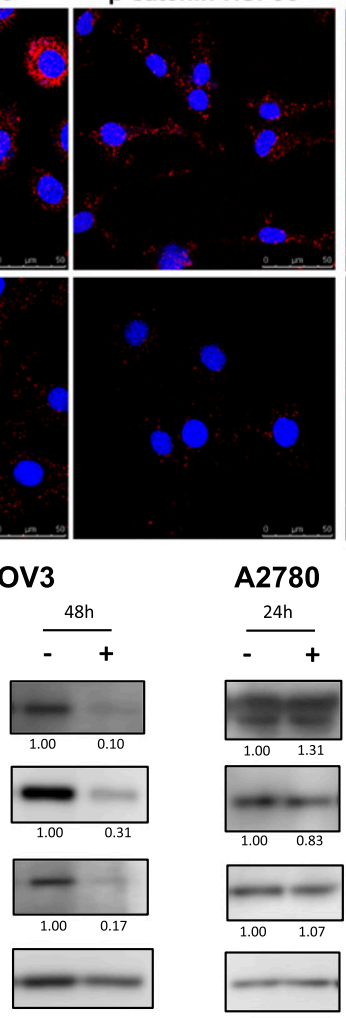

A2780
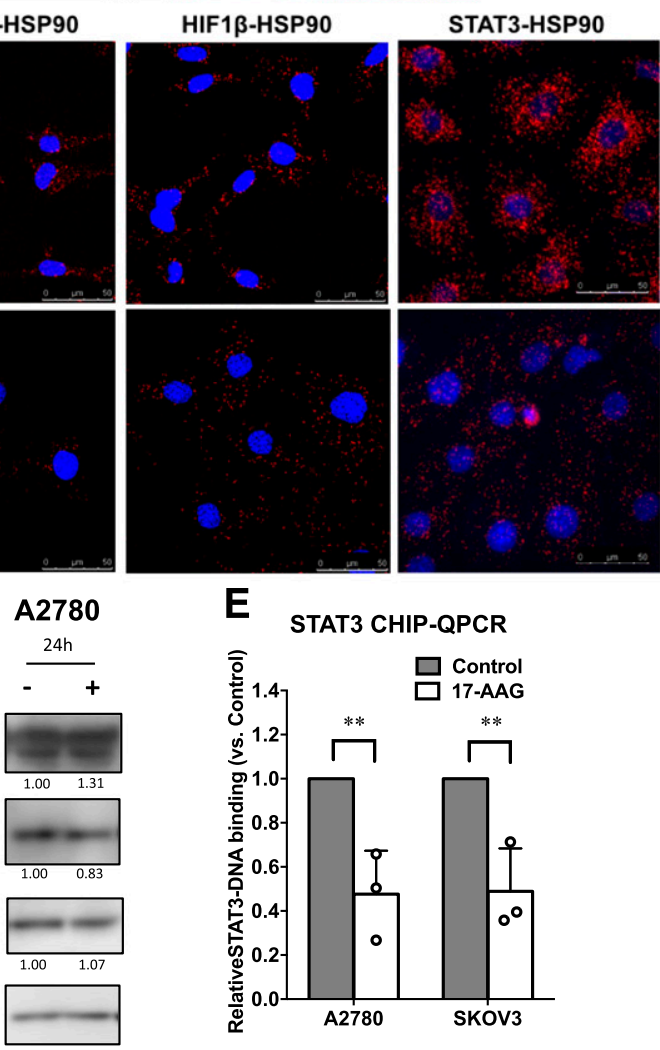

E
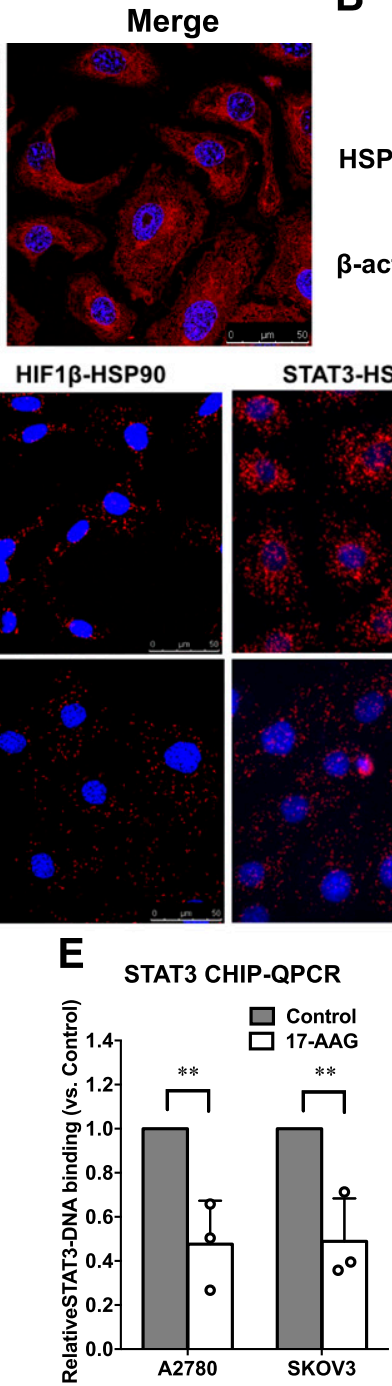

STAT3-HSP90

B

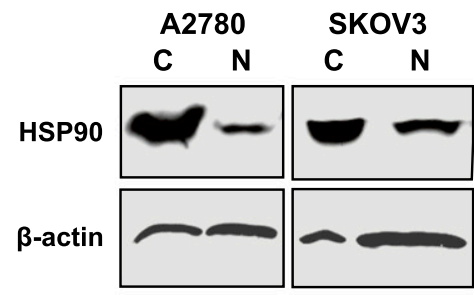

年

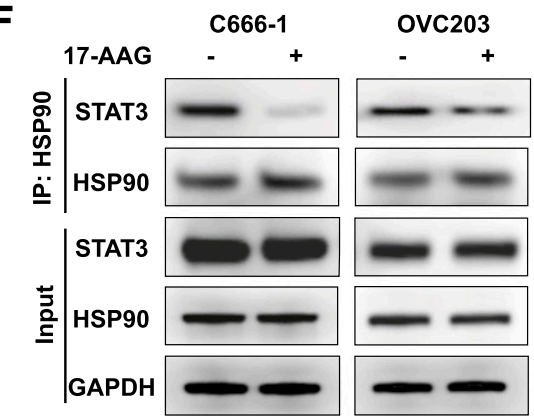

Fig. 3. Effects of HSP90 inhibition on Hsp90-STAT3 interaction and STAT3 transcriptional activity. (A) Representative confocal immunofluorescence images of HSP90 in SKOV3 cells. (B) Endogenous HSP90 protein in the cytoplasmic (C) and nuclear (N) fractions of A2780 and SKOV3 cells. $\beta$-actin is the loading control. (C) Representative images of PLA showing HSP90 interactions with HIF- $1 \alpha$, HIF- $1 \beta, \beta$-catenin, and STAT3 in SKOV3 cells treated with DMSO (control) or $0.5 \mu \mathrm{M}$ 17-AAG for 24 hours. (D) Western blot showing the protein levels of $\beta$-catenin, STAT3, and HIF-1 $\alpha$ in SKOV3 cells treated with DMSO or $0.5 \mu \mathrm{M}$ 17-AAG for 24 or 48 hours, and in A2780 cells treated with DMSO or $0.5 \mu \mathrm{M} 17$-AAG for 24 hours. (E) Binding of STAT3 to Twist1 promoter DNA measured by ChIP assay. Results are quantified with quantitative polymerase chain reaction (qPCR) and expressed as the amount of binding DNA relative to total input chromatin and normalized to control that was treated with DMSO. Values (mean \pm S.D.) represent three independent experiments $(n=3)$, each performed in triplicate. Two-way ANOVA test was followed by Tukey's honestly significant difference test. $* * P<0.005$. (F) Coimmunoprecipitation assays of STAT3 and HSP90. Nasopharyngeal carcinoma cell line C666-1 and the patient-derived serous epithelial ovarian cancer cell line OVC203 were treated with $0.5 \mu \mathrm{M}$ 17-AAG for 8 hours. Control groups were treated with $0.1 \%$ DMSO.

two inhibitors of STAT3, NSC74859 and nifuroxazide, to determine how STAT3 inhibition affects the ability of HSP90 to induce TWIST1 transcription. NSC74859 is a potent selective inhibitor blocking STAT3 DNA-binding activity (Siddiquee et al., 2007). Nifuroxazide suppresses the transcriptional activity of STAT1, 3, and 5 (Shi et al., 2017). Nifuroxazide has been shown to 
A

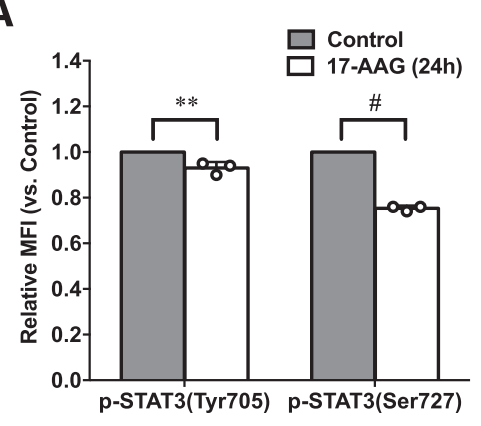

B

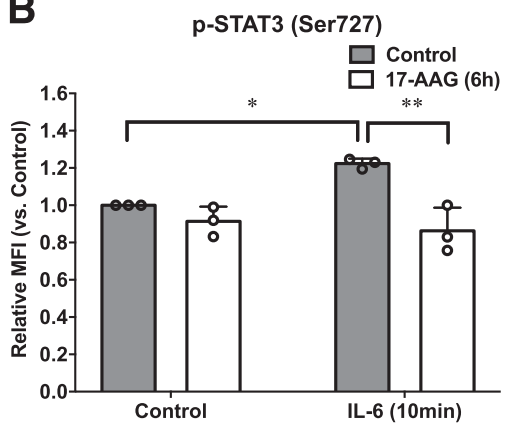

C

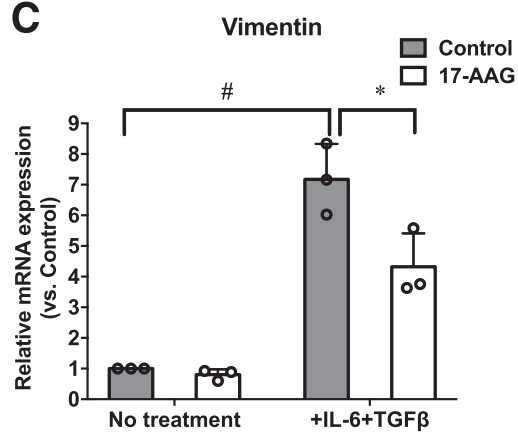

D
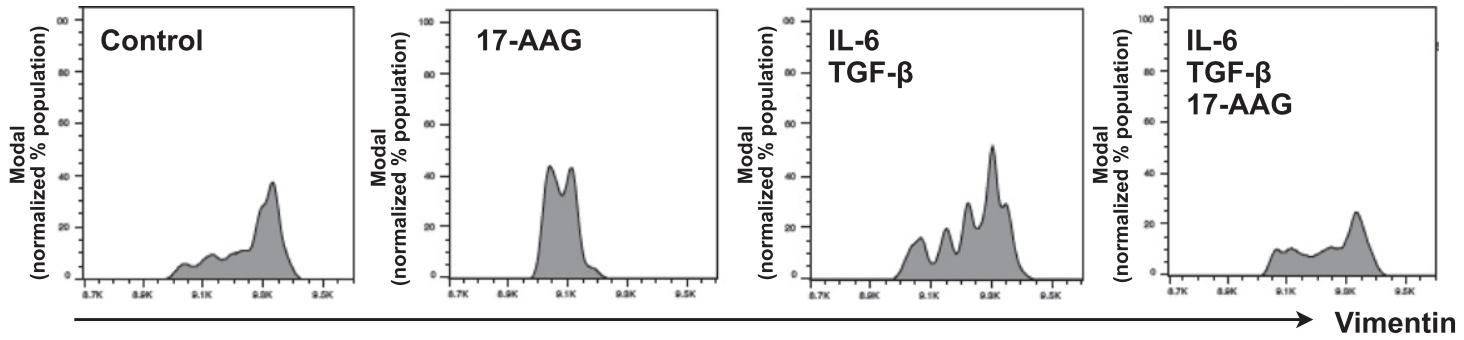

E

A498
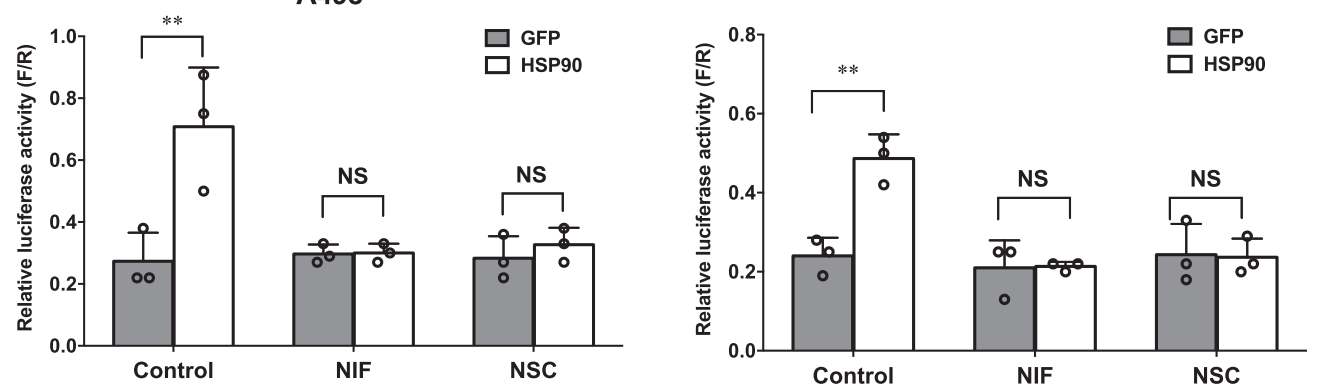

C666-1
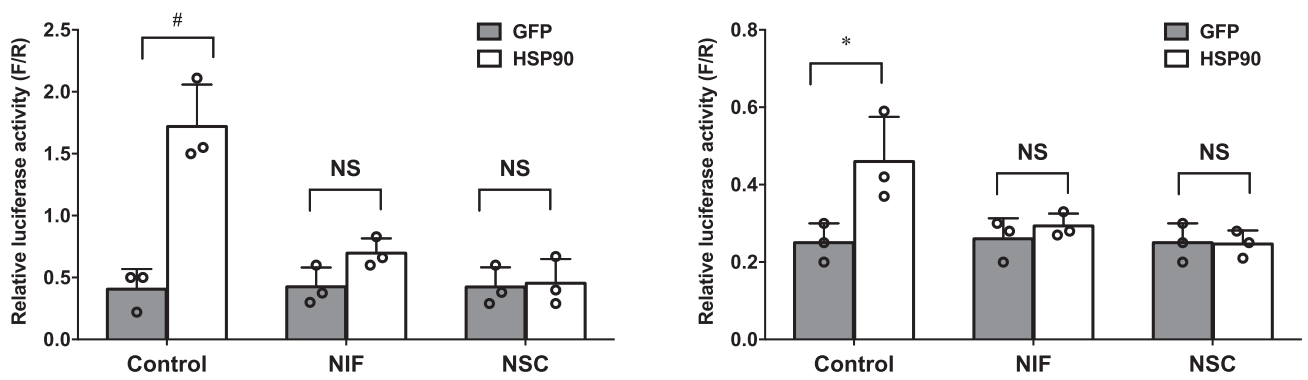

CNE1

Fig. 4. HSP90 cooperates with STAT3 to promote TWIST1 transcription. (A) Levels of p-STAT3 ${ }^{\text {Tyr705 }}$ and p-STAT3 ${ }^{\text {Ser727 }}$ in SKOV3 cells. Cells were treated with DMSO or $0.5 \mu \mathrm{M}$ 17-AAG for 24 hours. Values shown are the mean fluorescent intensity (MFI) normalized to the control measured by a beadbased assay. (B) p-STAT3 ${ }^{\text {Ser727 }}$ levels in SKOV3 cells treated with DMSO or $0.5 \mu \mathrm{M}$ 17- AAG for 6 hours or $1 \mathrm{nM}$ IL-6 for 10 minutes, or both in combination. (C) Vimentin mRNA expression in SKOV3 cells treated with DMSO or $0.5 \mu$ M 17-AAG for 24 hours, in the presence or absence of $1 \mathrm{nM}$ IL-6 in combination with $0.1 \mathrm{nM}$ transforming growth factor $\beta$ (TGF- $\beta$ ) for 14 days. (D) Vimentin protein levels in SKOV3 cells measured by flow cytometry. Cells received DMSO or a combination of $1 \mathrm{nM}$ IL- 6 and $0.1 \mathrm{nM}$ TGF- $\beta$ for 14 days, in the presence or absence of $0.5 \mu \mathrm{M} 17$-AAG in the last 24 hours of the 14-day treatment duration. The $y$-axis represents the percentage of cells. The $x$-axis represents fluorescent intensity that indicates vimentin expression level. (E) TWIST1 promoter activity in cancer cell lines transiently overexpressing GFP or Hsp90. Cells were treated with DMSO, $3 \mu$ M nifuroxazide (NIF) or $50 \mu \mathrm{M}$ NSC74859 (NSC) for 24 hours. Twist1 promoter activity was quantified as firefly luminescence relative to Renilla luminescence internal control. Values (mean \pm S.D.) represent three independent experiments $(n=3)$, each performed in triplicate. One-way ANOVA test was followed by Tukey's honestly significant difference test. ${ }^{\#} P<0.0001 ;{ }^{* *} P<0.005 ;{ }^{*} P<0.05$. NS, not significant.

inhibit tumor growth and metastasis in vivo by inhibiting STAT3 activity (Yang et al., 2015).

We overexpressed HSP90 by transient transfection to activate TWIST1 transcription in four cancer cell lines: A498, RCC4, C666-1, and CNE1. We inhibited STAT3 using NSC74859 and nifuroxazide and found that both inhibitors suppressed the activation of the TWIST1 promoter by HSP90 (Fig. 4E). These results support our hypothesis that HSP90 promotes TWIST1 transcription through the activation of STAT3.

The HSP90/STAT3/TWIST1 Pathway in PatientDerived Cancer Cell Lines and Tumor Samples. Next, we tested whether the inhibition of HSP90 suppressed TWIST1 
in patient-derived cells isolated from epithelial ovarian cancer samples of three patients. First, we observed that 17-AAG treatment significantly inhibited TWIST1 mRNA expression in all three patient-derived cancer cell lines (Fig. 5A). Second, we observed that 17-AAG significantly decreased TWIST1 promoter activity in the patient-derived ovarian cancer cell lines using luciferase reporter assays (Fig. 5B).

We tested the hypothesis that HSP90 interacts with STAT3 to regulate TWIST1 mRNA expression and EMT by demonstrating the HSP90-STAT3 interaction in cultured cancer cell lines; however, this interaction has not been observed in human tissues in situ. Therefore, we performed PLA staining to visualize the HSP90-STAT3 interaction in tumor samples.
We detected positive PLA staining, indicating the HSP90STAT3 interaction in approximately one-half of the 81 ovarian cancer tissue samples in a tissue array containing 1-mmdiameter tissue cores from 63 cases of serous type, 12 cases of mucinous type, five cases of clear cell type, and one endometrioid adenocarcinoma (Supplemental Figs. 6 and 7A). The distinct dotted signals from PLA staining were evident in the samples scored as medium or high. In contrast, the tissues scored as low showed very low or no PLA staining (Fig. 5C; Supplemental Fig. 7B). In the 57 cases of serous ovarian adenocarcinomas, the percentages of PLA staining in high, medium, and low samples were $34.6 \%, 15.4 \%$, and $50.0 \%$ in stage I tumors; $38.5 \%, 15.4 \%$, and $46.2 \%$ in stage II tumors;
A

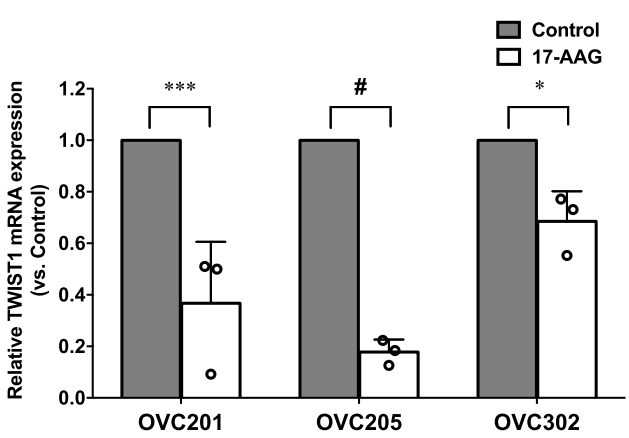

C
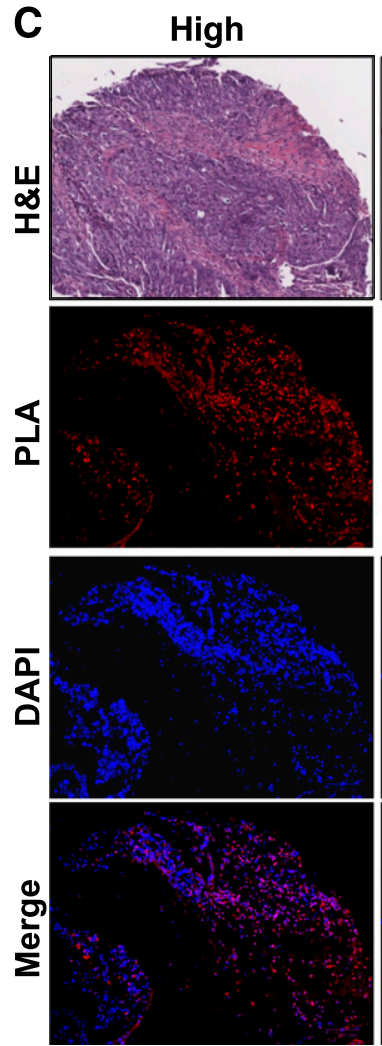

Medium
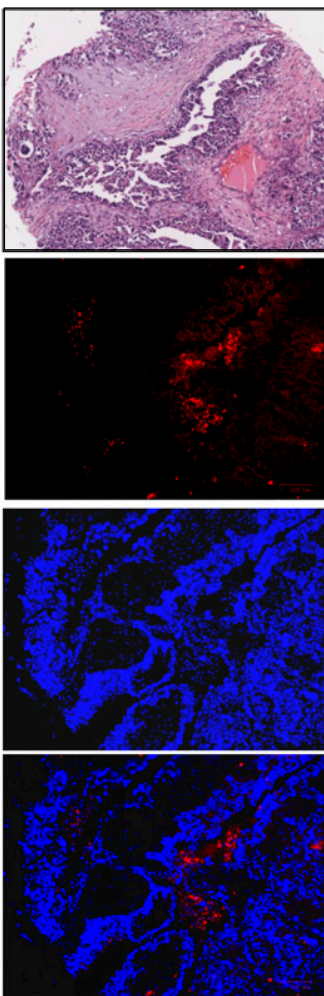

B

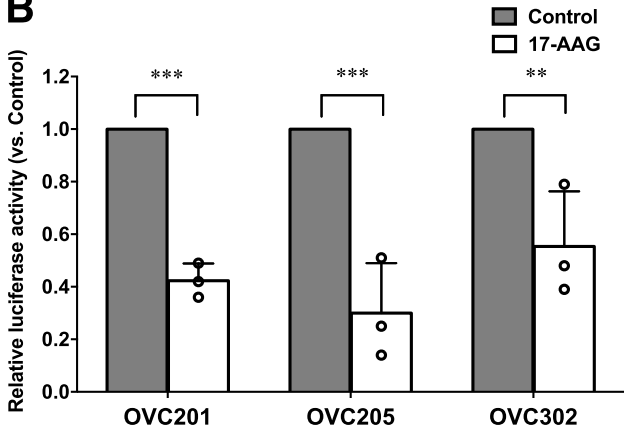

D
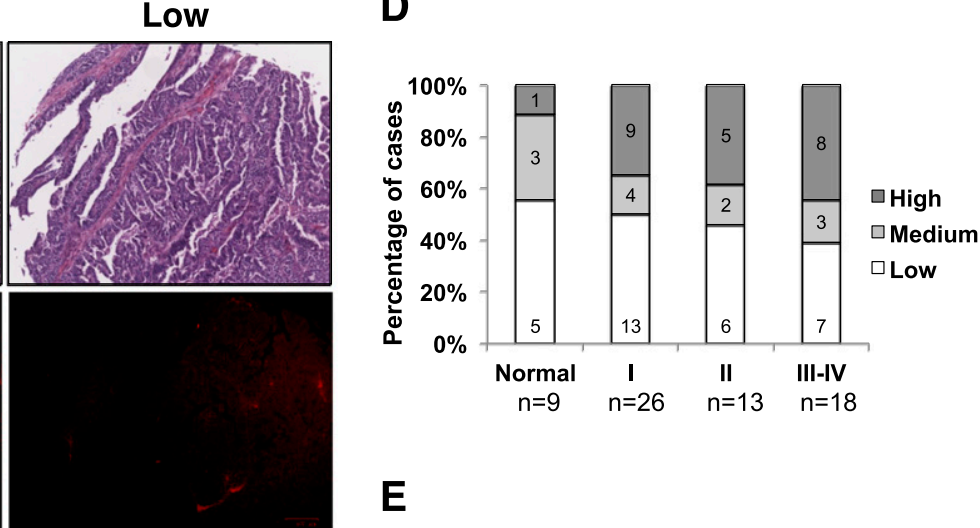

$\mathbf{E}$

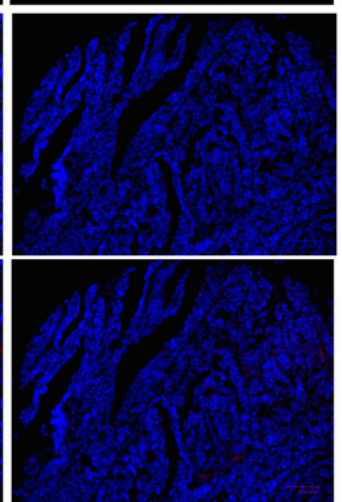

Fig. 5. HSP90/STAT3/TWIST1 pathway in patient-derived cancer cells and tumor samples. (A) TWIST1 mRNA levels were determined by quantitative polymerase chain reaction in epithelial ovarian cancer cells derived from patient tumor samples. Cells were treated with DMSO as control or $0.5 \mu \mathrm{M}$ 17-AAG for 24 hours. GAPDH was used as the reference gene and the mRNA expression levels were normalized to the control. (B) Twist1 promoter activity determined by luciferase reporter assays in patient-derived epithelial ovarian cancer cells treated with DMSO or 0.5 $\mu$ M $17-\mathrm{AAG}$ for 24 hours. (A and B) Two-way ANOVA test was followed by Tukey's honestly significant difference test. ${ }^{*} P<0.0001 ; * * * P<0.0005 ; * * P<0.005 ; * P<0.05$, (C) Representative images of in situ PLA performed using antibodies specific for HSP90 and STAT3 in ovarian tumor tissues. Red, PLA staining; blue, 4,6-diamidino-2-phenylindole (DAPI). (D) HSP90-STAT3 PLA staining results of serous ovarian cancer samples. The number of cases in each category is labeled inside the bars. (E) Schematic model. 
and $53.3 \%, 20.0 \%$, and $46.7 \%$ in stage III and IV tumors, respectively (Fig. 5D). One of the nine normal control ovarian tissues showed high PLA staining, and three showed medium levels of staining. The fraction of tumors with positive PLA staining was higher in the stage III and IV tumors than the early stage and normal tissues. Positive PLA staining was detected in about one-half of the clear cell-type (3/5) and mucinous-type ovarian cancer samples (6/12) (see the result summary and representative staining images in Supplemental Fig. 7).

Additionally, we assessed the HSP90-STAT3 interaction by PLA staining in four nasopharynx tissue samples. The two inflammatory nasopharyngeal tissues showed low or medium levels of PLA staining. The two cases of nasopharyngeal carcinomas showed medium or high levels of positive PLA staining (Supplemental Fig. 8).

Taken together, the inhibition of HSP90 by 17-AAG suppressed TWIST1 transcription in ovarian cancer cells derived from clinical samples. The interaction between HSP90 and STAT3 was visualized in cancer cell lines and tumor tissue samples of epithelial ovarian, renal clear cell, and nasopharyngeal cancers. The enhanced interaction between HSP90 and STAT3 may be associated with tumorigenesis and the progression of cancers. However, further analysis of large cohorts of tumor samples in combination with other molecular markers is required to determine the value of the HSP90/STAT3/TWIST1 pathway as a prognostic marker or therapeutic target.

\section{Discussion}

HSP90 has been identified as a potential driver of tumorigenesis given its roles in maintaining the function and structural integrity of various oncogenic proteins (Creagh et al., 2000; Barrott and Haystead, 2013). The inhibition of HSP90 inhibits the abundance and/or ability of oncoproteins to drive cell invasion and migration. However, the mechanisms by which HSP90 regulates such oncoproteins have not been explored in depth. Here, we report that the inhibition of HSP90 suppresses the promoter activity and expression of TWIST1. In addition, we demonstrate that the molecular mechanism of HSP90-regulated TWIST1 transcription involves STAT3.

Our findings suggest that HSP90 interacts with transcription factors both in the nuclei and cytoplasm to promote their activity. Although most of the known molecular actions are those of cytoplasmic HSP90, the functions of HSP90 in the nucleus are beginning to be recognized, particularly transcriptional regulation and gene expression.

In the nucleus, HSP90 has been found to localize near coding and noncoding gene promoters, where it stabilizes the negative elongation factor complex and maintains RNA polymerase II pausing (Sawarkar et al., 2012; Khurana and Bhattacharyya, 2015). HSP90 is also thought to be involved in the disassembly of regulatory complexes consisting of hormone-bound intracellular receptors (Freeman and Yamamoto, 2002). Our data and other studies support that HSP90 can directly interact with transcription factors to modulate transcription. For example, HSP90 binds to steroid hormone receptors to facilitate folding into a high-affinity hormone-binding structure (Nemoto et al., 1993). The interaction of HSP90 with transcription factors also enables nuclear transport of transcription factors and facilitates their subsequent binding to DNA (Kazlauskas et al., 2001; Echeverría et al., 2009; Galigniana et al., 2010). We demonstrate that HSP90 interacts with STAT3 in both the cytoplasm and nucleus to mediate TWIST1 expression (see the schematic model in Fig. 5E). Our study provides new evidence that nuclear HSP90 plays an important role in regulating gene transcription.

The inhibition of HSP90 suppresses cancer cell invasion and metastasis (McCready et al., 2014; White et al., 2016). HSP90 has been speculated to be an important player in the EMT process. However, previous findings have focused on extracellular or secreted HSP90 that can initiate EMT by stimulating the expression of EMT genes (Hance et al., 2012; Nolan et al., 2015). The roles of intracellular HSP90 in regulating EMT remain obscure. We show that direct interaction between HSP90 and STAT3 is essential for the activation of TWIST1 transcription. HSP90 also directly interacts with HIF-1 $\alpha$ and $\beta$-catenin in the cytoplasm and nuclei of cancer cells. Our data suggest the role of intracellular HSP90 as a regulator of EMT.

Initially, we selected eight transcriptional factors that have been identified as TWIST1 regulators and the clients of HSP90, including $\beta$-catenin, HIF- $1 \alpha$, HIF- $1 \beta$, STAT3, STAT5, STAT6, N-Myc, and MyoD1. We prioritized our investigation on STAT3 in this study due to several reasons. First, western blot results showed that STAT5, STAT6, N-Myc, and MyoD1 proteins were undetectable in ovarian cancer cell lines (Fig. 2A), which led us to focus on $\beta$-catenin, HIF- $1 \alpha$, HIF- $1 \beta$, and STAT3. Second, we performed ChIP assays for $\beta$-catenin, HIF- $1 \alpha$, HIF-1 $\beta$, and STAT3. The bindings of $\beta$-catenin, HIF- $1 \alpha$, and HIF- $1 \beta$ to TWIST 1 promoter were not significantly changed by 17-AAG treatment after 24 hours (data not shown). The binding of STAT3 to TWIST1 promoter was reduced significantly by 17-AAG treatment for 24 hours (Fig. 3E). Finally, STAT3 is a vital pro-oncogenic transcription factor that is constitutively activated and phosphorylated in many cancers (Qin et al., 2008). The HSP90-regulated STAT3 activity is likely to play a critical role in tumor progression and drug resistance in many cancers, particularly considering the function of STAT3 in promoting EMT.

HSP90 was previously reported to interact with and stabilize STAT3 into an active conformation favorable for phosphorylation and dimerization and to promote the nuclear localization of STAT3 (Bocchini et al., 2014). Additionally, the HSP90-STAT3 interaction enhances STAT3 activity by promoting $\mathrm{Ser}^{727}$ phosphorylation. The IL-6/JAK/STAT3 signaling pathway is well known to induce EMT in various cancers, and STAT3 has been shown to transcriptionally activate TWIST1 (Colomiere et al., 2009; Sullivan et al., 2009; Chen et al., 2015). Our data identified that the intracellular HSP90 and STAT3 interaction enables STAT3 to bind to the TWIST1 promoter. A potential therapeutic strategy that could be derived from these findings is that inhibitors of HSP90 or STAT3 may suppress this process and inhibit EMT, potentially slowing down tumor growth, invasion, dissemination, metastasis, and/or drug resistance.

Recent genomic profiling studies have revealed that A2780 and SKOV3 do not represent the most common type of ovarian cancer, high-grade serous ovarian carcinoma. They are more likely to be endometroid or clear cell carcinoma cells. On the one hand, we used these cell lines in this study because they have been the most commonly used ovarian cancer cell lines in studying cancer biology and evaluating new therapeutic agents in vitro and in vivo. Their genome, transcriptome, and proteome 
have been characterized in detail. On the other hand, our hypothesis is that the interaction of HSP90 and STAT3 promotes TWIST1 expression and EMT, which is not limited to a certain histotype of ovarian cancer; it can be applied to many types of cancer in which HSP90 and STAT3 are highly activated. To mitigate the limitation of these cell lines and strengthen our hypothesis, in our study we also included four other ovarian cancer cell lines that were derived from patients with serous-type epithelial ovarian cancer.

The three cancer types that were investigated in this study are epithelial ovarian, clear cell renal, and nasopharyngeal cancers. When diagnosed at advanced stages, epithelial ovarian cancer patients usually receive debulking surgery and platinum/paclitaxel chemotherapy. The advanced clear cell renal cancer patients are treated with cytoreductive nephrectomy followed by tyrosine kinase inhibitors, such as sunitinib, sarafenib, and pazopanib. Inhibitors of mammalian target of rapamycin kinase and immune checkpoint blockade have also been approved as treatments for advanced renal cancer. For nasopharyngeal carcinoma, surgery is only occasionally performed. The majority of patients with nasopharyngeal carcinoma are treated with radiation therapy or chemoradiation, which is chemotherapy given along with radiation. The most frequently used chemotherapy drugs for nasopharyngeal carcinoma are cisplatin, carboplatin, and fluorouracil.

These tumor types differ anatomically, genetically, and phenotypically, which explains the differences in treatment strategies. However, like many other cancer types, these cancer cells commonly maintain high levels of HSP90 and/or constitutively activated STAT3 (Huang et al., 2000; Guo et al., 2009; Cheng et al., 2018). TWIST1 and EMT have been shown to play key roles in the progression and drug resistance in these cancers (Davidowitz et al., 2014; Zhuo et al., 2014; Singla et al., 2018). Our work provides insight into how Hsp90 inhibitors could suppress STAT3/TWIST1 signaling and EMT in these cancer cells. This will hopefully pave the way for new therapeutic opportunities to target EMT and metastasis using HSP90 inhibitors.

\section{Acknowledgments}

We thank Dr. Carlotta A. Glackin (City of Hope Beckman Research Institute, Duarte, CA) for providing the Twist1 1-kilobase promoter luciferase reporter plasmid, Dr. Anirban Mitra (Indiana University School of Medicine, Indianapolis, IN) for providing the OVCAR8 cells, Dr. Gil Mor (Yale University School of Medicine) for providing the A2780 cells and patient-derived ovarian cancer cell lines, and Jamie Goodner-Bingham for participating in part of the experiment design.

\section{Authorship Contributions}

Participated in research design: Chong, Kang, Garofalo, Liang, Yang-Hartwich.

Conducted experiments: Chong, Kang, Garofalo, Ueno, Madarikan, Pitruzzello, Tsai, Hartwich.

Performed data analysis: Chong, Kang, Garofalo, Liang, Hartwich, Yang-Hartwich.

Wrote or contributed to the writing of the manuscript: Chong, Cady, Hartwich, Shuch, Yang-Hartwich.

\section{References}

Bahmad HF, Cheaito K, Chalhoub RM, Hadadeh O, Monzer A, Ballout F, El-Hajj A, Mukherji D, Liu YN, Daoud G, et al. (2018) Sphere-formation assay: three-dimensional in vitro culturing of prostate cancer stem/progenitor sphere-forming cells. Front Oncol 8:347.

Barrott JJ and Haystead TA (2013) Hsp90, an unlikely ally in the war on cancer FEBS J 280:1381-1396.
Bocchini CE, Kasembeli MM, Roh SH, and Tweardy DJ (2014) Contribution of chaperones to STAT pathway signaling. JAK-STAT 3:e970459.

Chen J, Gong C, Mao H, Li Z, Fang Z, Chen Q, Lin M, Jiang X, Hu Y, Wang W, et al. (2018) E2F1/SP3/STAT6 axis is required for IL-4-induced epithelial-mesenchymal transition of colorectal cancer cells. Int J Oncol 53:567-578.

Chen W, Gao Q, Han S, Pan F, and Fan W (2015) The CCL2/CCR2 axis enhances IL-6-induced epithelial-mesenchymal transition by cooperatively activating STAT3-Twist signaling. Tumour Biol 36:973-981.

Cheng GZ, Zhang WZ, Sun M, Wang Q, Coppola D, Mansour M, Xu LM, Costanzo C, Cheng JQ, and Wang LH (2008) Twist is transcriptionally induced by activation of STAT3 and mediates STAT3 oncogenic function. J Biol Chem 283:14665-14673.

Cheng JZ, Chen JJ, Xue K, Wang ZG, and Yu D (2018) Clinicopathologic and prognostic significance of VEGF, JAK2 and STAT3 in patients with nasopharyngeal carcinoma. Cancer Cell Int 18:110.

Colomiere M, Ward AC, Riley C, Trenerry MK, Cameron-Smith D, Findlay J, Ackland L, and Ahmed N (2009) Cross talk of signals between EGFR and IL-6R through JAK2/STAT3 mediate epithelial-mesenchymal transition in ovarian carcinomas. Br J Cancer 100:134-144.

Creagh EM, Sheehan D, and Cotter TG (2000) Heat shock proteins-modulators of apoptosis in tumour cells. Leukemia 14:1161-1173.

Davidowitz RA, Selfors LM, Iwanicki MP, Elias KM, Karst A, Piao H, Ince TA, Drage MG, Dering J, Konecny GE, et al. (2014) Mesenchymal gene program-expressing ovarian cancer spheroids exhibit enhanced mesothelial clearance. J Clin Invest 124:2611-2625.

Diehl MC, Idowu MO, Kimmelshue K, York TP, Elmore LW, and Holt SE (2009) Elevated expression of nuclear Hsp90 in invasive breast tumors. Cancer Biol Ther 8:1952-1961.

Echeverría PC, Mazaira G, Erlejman A, Gomez-Sanchez C, Piwien Pilipuk G, and Galigniana MD (2009) Nuclear import of the glucocorticoid receptor-hsp90 complex through the nuclear pore complex is mediated by its interaction with Nup62 and importin $\beta$. Mol Cell Biol 29:4788-4797.

Freeman BC and Yamamoto KR (2002) Disassembly of transcriptional regulatory complexes by molecular chaperones. Science 296:2232-2235.

Galigniana MD, Erlejman AG, Monte M, Gomez-Sanchez C and Piwien-Pilipuk G (2010) The hsp90-FKBP52 complex links the mineralocorticoid receptor to motor proteins and persists bound to the receptor in early nuclear events. Mol Cell Biol 30:1285-1298.

Guo C, Yang G, Khun K, Kong X, Levy D, Lee P, and Melamed J (2009) Activation of Stat3 in renal tumors. Am J Transl Res 1:283-290.

Hance MW, Dole K, Gopal U, Bohonowych JE, Jezierska-Drutel A, Neumann CA, Liu H, Garraway IP, and Isaacs JS (2012) Secreted Hsp90 is a novel regulator of the epithelial to mesenchymal transition (EMT) in prostate cancer. $J$ Biol Chem 287: 37732-37744.

Hartl FU and Hayer-Hartl M (2009) Converging concepts of protein folding in vitro and in vivo. Nat Struct Mol Biol 16:574-581.

Howe LR, Watanabe O, Leonard J, and Brown AM (2003) Twist is up-regulated in response to Wnt1 and inhibits mouse mammary cell differentiation. Cancer Res 63: $1906-1913$

Huang M, Page C, Reynolds RK, and Lin J (2000) Constitutive activation of stat 3 oncogene product in human ovarian carcinoma cells. Gynecol Oncol 79:67-73.

Jolly C and Morimoto RI (2000) Role of the heat shock response and molecular chaperones in oncogenesis and cell death. J Natl Cancer Inst 92:1564-1572.

Kazlauskas A, Sundström S, Poellinger L, and Pongratz I (2001) The hsp90 chaperone complex regulates intracellular localization of the dioxin receptor. Mol Cell Biol 21: 2594-2607.

Khurana N and Bhattacharyya S (2015) Hsp90, the concertmaster: tuning transcription. Front Oncol 5:100.

Koutalianos D, Koutsoulidou A, Mastroyiannopoulos NP, Furling D, and Phylactou LA (2015) MyoD transcription factor induces myogenesis by inhibiting Twist-1 through miR-206. J Cell Sci 128:3631-3645.

Liu RY, Zeng Y, Lei Z, Wang L, Yang H, Liu Z, Zhao J, and Zhang HT (2014) JAK/STAT3 signaling is required for TGF- $\beta$-induced epithelial-mesenchymal transition in lung cancer cells. Int $J$ Oncol 44:1643-1651.

Luo W, Sun W, Taldone T, Rodina A, and Chiosis G (2010) Heat shock protein 90 in neurodegenerative diseases. Mol Neurodegener 5:24.

McClellan AJ, Tam S, Kaganovich D, and Frydman J (2005) Protein quality control: chaperones culling corrupt conformations. Nat Cell Biol 7:736-741.

McClellan AJ, Xia Y, Deutschbauer AM, Davis RW, Gerstein M, and Frydman J (2007) Diverse cellular functions of the Hsp90 molecular chaperone uncovered using systems approaches. Cell 131:121-135.

McCready J, Wong DS, Burlison JA, Ying W, and Jay DG (2014) An impermeant ganetespib analog inhibits extracellular Hsp90-mediated cancer cell migration that involves lysyl oxidase 2-like protein. Cancers (Basel) 6:1031-1046.

Nemoto T, Ohara-Nemoto Y, Sato N, and Ota M (1993) Dual roles of 90-kDa heat shock protein in the function of the mineralocorticoid receptor. $J$ Biochem 113: $769-775$.

Nolan KD, Franco OE, Hance MW, Hayward SW, and Isaacs JS (2015) Tumor-secreted Hsp90 subverts polycomb function to drive prostate tumor growth and invasion. J Biol Chem 290:8271-8282

Qin HR, Kim HJ, Kim JY, Hurt EM, Klarmann GJ, Kawasaki BT, Duhagon Serrat MA and Farrar WL (2008) Activation of signal transducer and activator of transcription 3 through a phosphomimetic serine 727 promotes prostate tumorigenesis independent of tyrosine 705 phosphorylation. Cancer Res 68:7736-7741.

Qin Q, Xu Y, He T, Qin C, and Xu J (2012) Normal and disease-related biological functions of Twist1 and underlying molecular mechanisms. Cell Res 22:90-106.

Sato N, Yamamoto T, Sekine Y, Yumioka T, Junicho A, Fuse H, and Matsuda T (2003) Involvement of heat-shock protein 90 in the interleukin-6-mediated signaling pathway through STAT3. Biochem Biophys Res Commun 300:847-852.

Sawarkar R, Sievers C, and Paro R (2012) Hsp90 globally targets paused RNA polymerase to regulate gene expression in response to environmental stimuli. Cell 149:807-818. 
Schuringa JJ, Wierenga AT, Kruijer W, and Vellenga E (2000) Constitutive Stat3, Tyr705, and Ser727 phosphorylation in acute myeloid leukemia cells caused by the autocrine secretion of interleukin-6. Blood 95:3765-3770.

Selmi A, de Saint-Jean M, Jallas AC, Garin E, Hogarty MD, Bénard J, Puisieux A, Marabelle A, and Valsesia-Wittmann S (2015) TWIST1 is a direct transcriptional target of $M Y C N$ and $M Y C$ in neuroblastoma. Cancer Lett 357:412-418.

Shi L, Zheng H, Hu W, Zhou B, Dai X, Zhang Y, Liu Z, Wu X, Zhao C, and Liang G (2017) Niclosamide inhibition of STAT3 synergizes with erlotinib in human colon cancer. OncoTargets Ther 10:1767-1776.

Siddiquee K, Zhang S, Guida WC, Blaskovich MA, Greedy B, Lawrence HR, Yip ML, Jove R, McLaughlin MM, Lawrence NJ, et al. (2007) Selective chemical probe inhibitor of Stat3, identified through structure-based virtual screening, induces antitumor activity. Proc Natl Acad Sci USA 104:7391-7396

Singla M, Kumar A, Bal A, Sarkar S, and Bhattacharyya S (2018) Epithelial to mesenchymal transition induces stem cell like phenotype in renal cell carcinoma cells. Cancer Cell Int 18:57.

Su JM, Hsu YY, Lin P, and Chang H (2016) Nuclear accumulation of heat-shock protein 90 is associated with poor survival and metastasis in patients with nonsmall cell lung cancer. Anticancer Res 36:2197-2203.

Sullivan NJ, Sasser AK, Axel AE, Vesuna F, Raman V, Ramirez N, Oberyszyn TM, and Hall BM (2009) Interleukin-6 induces an epithelial-mesenchymal transition phenotype in human breast cancer cells. Oncogene 28:2940-2947.

Wang H, Lu M, Yao M, and Zhu W (2016) Effects of treatment with an Hsp90 inhibitor in tumors based on 15 phase II clinical trials. Mol Clin Oncol 5 326-334

White PT, Subramanian C, Zhu Q, Zhang H, Zhao H, Gallagher R, Timmermann BN, Blagg BS, and Cohen MS (2016) Novel HSP90 inhibitors effectively target functions of thyroid cancer stem cell preventing migration and invasion. Surgery 159:142-151.
Wu P, Wu D, Zhao L, Huang L, Shen G, Huang J, and Chai Y (2016) Prognostic role of STAT3 in solid tumors: a systematic review and meta-analysis. Oncotarget 7:19863-19883.

Yang F, Hu M, Lei Q, Xia Y, Zhu Y, Song X, Li Y, Jie H, Liu C, Xiong Y, et al. (2015) Nifuroxazide induces apoptosis and impairs pulmonary metastasis in breast cancer model. Cell Death Dis 6:e1701.

Yang J, Mani SA, Donaher JL, Ramaswamy S, Itzykson RA, Come C, Savagner P, Gitelman I, Richardson A, and Weinberg RA (2004) Twist, a master regulator of morphogenesis, plays an essential role in tumor metastasis. Cell 117:927-939.

Yang MH, Wu MZ, Chiou SH, Chen PM, Chang SY, Liu CJ, Teng SC, and Wu KJ (2008) Direct regulation of TWIST by HIF-1 $\alpha$ promotes metastasis. Nat Cell Biol 10:295-305.

Zhao C, Wang Q, Wang B, Sun Q, He Z, Hong J, Kuehn F, Liu E, and Zhang Z (2017) IGF-1 induces the epithelial-mesenchymal transition via Stat5 in hepatocellular carcinoma. Oncotarget 8:111922-111930.

Zhao R, Davey M, Hsu YC, Kaplanek P, Tong A, Parsons AB, Krogan N, Cagney G, Mai $\mathrm{D}$, Greenblatt J, et al. (2005) Navigating the chaperone network: an integrative map of physical and genetic interactions mediated by the hsp90 chaperone. Cell 120:715-727.

Zhuo X, Chang A, Huang C, Yang L, Xiang Z, and Zhou Y (2014) Expression of TWIST, an inducer of epithelial-mesenchymal transition, in nasopharyngeal carcinoma and its clinical significance. Int J Clin Exp Pathol 7:8862-8868.

Address correspondence to: Dr. Yang Yang-Hartwich, Department of Obstetrics, Gynecology, and Reproductive Sciences, Yale School of Medicine, 375 Congress Avenue, LSOG209, New Haven, CT 06510. E-mail: yang.yang@ yale.edu 